\title{
Analysis and Effective Measures to Shorten Processing Time of Faults in Secondary Circuit
}

\author{
Zhu Ming, Zheng Wei, Zhao Lisong \\ Benxi Power Supply Company of National Grid Liaoning Electric Power Co., Ltd. \\ bxwl_650928@126.com
}

Keywords: secondary circuit, fault time, tools

\begin{abstract}
The condition of secondary circuit directly determines whether mechanism box can be electrically operated, and qualification of the maintenance of the whole equipment depends on whether electric operation is in place and meets the requirements. Therefore, maintenance of the secondary circuit is the beginning and essential part of the whole maintenance. According to the index requirement, the processing time of fault in secondary circuit $(\mathrm{T})$ and number of faults $(\mathrm{N})$ should meet $\mathrm{T} \leq 7 \mathrm{~N}-2(\mathrm{~N} \geq 1)$ and generally the time of secondary circuit maintenance should be no more than $10 \%$ of the entire maintenance time.
\end{abstract}

\section{Present Situation Investigation}

The complicated cables used in secondary circuit are prone to lead to frequent faults in the mechanism box of electrical equipment and the restored cable lines in the mechanism box become more confusing, resulting in unnecessary troubles for fault detection. Meanwhile, wrong contacts may be connected in the wiring process and it may directly leads to faults of no-phase or reverse phase, etc., imposing a risk for the stability of power system and sometimes even directly affecting the transmission time, resulting in both economic and reputation losses.

According to investigations, reasons for faults or inefficient maintenance in secondary circuit are mainly lack of professional training, absence of professional wiring tools, small volume of mechanism box itself, inconsistent manufacturers of replaced cable with the original one, nonmatching attachments from the manufacturers and bad weather, etc..

In the collection of statistics on the maintenance process of each mechanism box with faults, since the cable lines in the mechanism box is complicated with small components and the close contacts make it easily to find out wrong contacts or fault connects between adjacent contacts leading to loose contacts, it can be dealt for one time from fault checking, processing to test operation. The probability of no repeated checking and processing is around $70 \%$, which is not too high but can be improved.

As found in experiments, if the mechanism box with the same fault has a neat wiring, processing time of faults can be greatly reduced, it is easier to detect the contact position and cable routing, and even those faults needing wire changing can be processed easily and with a high success rate.

Therefore, although it is inevitable to cause faults by the multiple cables and complicated wiring in the mechanism box, poor quality of the cable itself, bad environment and temperature changes, etc., due to the long lasting maintenance time of wiring problems, low maintenance quality and efficiency can be improved and even avoided.

\section{Setting Objectives}

Shorten the entire processing time of faults in secondary circuit by $25 \%$.

\section{Analyzing the Reasons}

Two factors are obtained by analysis: 
Lack of professional training

Absence of professional wiring tools

\section{Confirming the Main Reason}

\section{Analyzing the Content of Main Reason}

Reason one: lack of professional training

Accumulated by solid theoretical knowledge and numerous experiences, substation equipment maintenance technology of the workers determines the speed and quality of maintenance. Currently, there is a polarized development in the ages of substation equipment maintenance workers and most of them are the elder workers dispatched from the grass-root positions of various power construction enterprises and recently graduated students, whose maintenance capacity differs greatly and hardly to be both good at theory and technical level.

Conclusion: The lack of professional trainings is the main reason.

Reason two: absence of professional wiring tools

Fault points can hardly be found if there is in secondary circuit but generally by screening many components. Majority of faults are not caused by single problems and wiring confusion makes it difficult to distinguish the connections between contacts and components. If it is equipment used for a long period, one can hardly recognize the blurred words on the connection cap and not to say determine the source of the wire, but to figure it out by check each wire according to the circuit diagram.

Conclusion: absence of professional wiring tools is the main reason.

\section{Confirming the Main Reason}

Main reasons are confirmed after analysis on the content and proportion of main reasons as following:

1) Absence of professional wiring tools

2) Lack of professional training

\section{Developing Countermeasures}

Evaluate each of the above main reasons comprehensively from the economic, technical and difficult levels and countermeasures are designed as follows:

(1)Absence of professional wiring tools: design tools and the model made are successful and easy to carry with and use. With the tools, processing time of faults each time is reduced by $25 \%$.

(2)Lack of professional training: arrange regular trainings and improve maintenance level. Processing time of faults in secondary circuit per capita reaches the standard, namely $\mathrm{T} \leq 7 \mathrm{~N}-2$ $(\mathrm{N} \geq 1)$.

\section{Implementation of Countermeasures}

\section{Design Tools}

\section{1) Principle}

Roll straightening under pressure principle is adopted in tools design. The figure on the right is the schematic diagram (guide ring 1; straightening drum 2; transport roller 3; straightening roller 4). The position of rollers is mainly used to form certain angle with the moving direction of straightened products. Two or three big ones are active pressure rollers and driven by a rally to rotate in the same direction; in the other end are several small ones functioning as the slave rollers driven by rotating rod or pipe friction to rotate. In order to achieve the pressure required by the cables, these small rollers should adjust positions forward or backward simultaneously or separately. Generally, the more the number of rollers is, the more accurate the straightened cables are. After being nipped by the roller, cables continue to make linear or rotary motions and impose compressing, bending and flattening deformations on the cable, achieving the objective of straightening. 


\section{2) Structure and Design Explanation}

The specialty of straightener for cables in secondary circuit lies in its shell. The shell is composed by folded upper and lower shells. There are two pairs of rollers in the same vertical plane inside the shell. Each pair of rollers is fixed by shafts onto the upper and lower shells and rollers are laminated with many corresponding electric raceways where entry and outlet ports are set.

The entry port is bell shaped to avoid destroy the insulation layer during the entry process.

In the position of corresponding fixed rollers in upper and lower shell bearings are set to ensure the rotation of shaft under the drive of rollers and cable output.

The shaft and the rollers are connected by key joint.

The rollers are made of Bakelite.

\section{3) Results of Implementation}

Faults in the experiment are set artificially. The main purpose is to prove that straightener for cables in secondary circuit can straighten cables effectively and that mechanism boxes of wiring with straightened cables are better the original ones both in wiring speed and liability.

The 1st, 2nd and 3rd mechanism box are wired by the same person. The average wiring speed with straightener is 23.3 minutes, while the average wiring speed without straightener is 33.3 minutes, with a 10 minutes lag than the former one.

Carry out faults detection and recovery in the mechanism boxes of the same type by the same person. The set faults are all that need replacing cables, such as cable line-broken, poor contact joints and so on. There are temporarily no settings of component damage faults. The average faults processing speed with straightener is about 6 minutes for each one, while that without straighteners is 8.5 minutes for each fault. Therefore, wiring after cable straightening can improve the wiring speed significantly, induce maintenance time and at the same time reduce repetitions in maintenance of equipment, thus reducing maintenance cost and increasing economic efficiency for the enterprise.

As can be seen from the data, processing time of each fault with straightener is shortened by $25 \%$ and reaches the set objective.

\section{Regular Trainings}

Carry out targeted trainings. Arrange lectures on safety procedures and technical skills, etc. for employees. Take theory as the basis and proceed from practices. Encourage the workers to learn while working and emphasis application. Strengthen the practical field operational capacity of workers and enable them to adapt to the work environment quickly and obtain various operational skills. Arrange interactive teaching and learning and encourage workers to propose questions for discussion and thus solve existing problems in work. Select workers that have been in the competition at and above provincial levels and encourage them to share the acquired new technologies, new processes and new requirements with their colleagues.

\section{Effect Examination}

The maintenance time of a mechanical box with no more than 3 faults is set as 20 minutes. After repeated experiments, the average processing time is reduced to 10 minutes, declining $50 \%$ from the former 20 minutes. Based on the processing time of secondary circuit and maintenance proportion, the average single interruption maintenance time is calculated as 2 hours, while the average yearly interruption maintenance of faults is 1 hour. Based on the entire running of a $10 \mathrm{KV}$ line, the average load of each line is calculated as about $2000 \mathrm{KV}$. The power supply loses in each maintenance is about 2000kwh; the total of labor cost saved and line loses is 1437 Yuan; assuming that there are 20 faults yearly on average, the cost saved a year is 28740 Yuan, which can help improve the economic efficiency.

\section{Conclusion}

Effective methods can be taken to reduce the maintenance time of secondary circuit and gradually improve the quality awareness, problem awareness and problem-solving ability of 
employees during the process, thus enhancing the quality of the staff, routinizing organization forms and contributing to the realization of quality assurance, technical complementary, knowledge sharing and team harmony. Meanwhile, they can also promote team building, cultivate the concepts of the staff to accept and promote modern management and improve further the comprehensive quality of the staff.

\section{References}

[1] Chen Shiqing. On the Condition-based Maintenance of Power Transformer [J]. Transformer.

[2] Lu Jizhi. On the Condition-based Maintenance and Development of Electric Transmission and Transformation Equipment[J ].Yunan Electric Power.

[3] Ma Jie. Carry out Equipment Condition-based Maintenance and Ensure the Safe Operation of Power Grid[J].Sci-Tech Information Development \& Economy.

[4] China Planning Press. Collaboration for the Construction and Acceptance Standard of Installation Project of Electrical Device [M].Beijing:China Planning Press.

[5] Chemistry Standardization for Power Plant Technical Committee of Ministry of Electric Power Industry. Collaboration for the Standards of Quality and Test Method of Oil Used for Electric Power [M]. China Standard Press. 